TRANSACTIONS OF THE

AMERICAN MATHEMATICAL SOCIETY

Volume 350, Number 3, March 1998, Pages 1129-1141

S 0002-9947(98)02097-2

\title{
ON THE EQUIVARIANT MORSE COMPLEX OF THE FREE LOOP SPACE OF A SURFACE
}

\author{
NANCY HINGSTON
}

\begin{abstract}
We prove two theorems about the equivariant topology of the free loop space of a surface. The first deals with the nondegenerate case and says that the "ordinary" Morse complex can be given an $O(2)$-action in such a way that it carries the $O(2)$-homotopy type of the free loop space. The second says that, in terms of topology, the iterates of an isolated degenerate closed geodesic "look like" the continuous limit of the iterates of a finite, fixed number of nondegenerate closed geodesics.
\end{abstract}

Let $M$ be a compact Riemannian manifold, and $\Lambda=\operatorname{Maps}\left(S^{1}, M\right)$ the free loop space of $M$. The energy function $E: \Lambda \rightarrow \mathbb{R}$ has as its critical points the closed geodesics on $M$. The group $O(2)$ of isometries of $S^{1}$ acts on $\Lambda$ leaving the energy function invariant. Morse theory allows one to describe the equivariant homotopy type of $\Lambda$ in terms of the closed geodesics on $M$ and their indices as critical points. We show that, when $M$ is a surface, this connection between topology and geometry is in some sense optimal, as direct as one could hope for. The first proposition deals with the nondegenerate case and gives an equivariant version of the Morse chain complex. The second says that, in terms of topology, the iterates of a degenerate closed geodesic can be resolved as the continuous limit of the iterates of a finite, fixed number of nondegenerate closed geodesics. These results are applied in [6], where we show that if $M$ is a two-sphere, the number $N(\ell)$ of closed geodesics of length $\leq \ell$ grows at least like the prime numbers, that is,

$$
\liminf N(\ell) \frac{\log (\ell)}{\ell}>0 .
$$

Proposition I is nice from a theoretical point of view (see the third remark below), but even more important from a practical point of view, as it allows one to do something which authors since Morse (see the last remark) have attempted unsuccessfully: to do Morse theory on the free loop space equivariantly without involving the lower strata (by the action of the stability group) of the negative bundle of a critical point. Proposition I is precisely what one needs in order to carry out equivariantly the usual geometric constructions (e.g. minimax) associated with Morse theory (see Corollaries 1, 2, 3). The effect of Proposition II in the above application is to reduce the degenerate to the nondegenerate case. The analog of Proposition II seems to be true for manifolds $M$ of higher dimension; however, as explained at the very end, we think it very unlikely that Proposition I holds in higher dimensions.

Received by the editors May 4, 1996

1991 Mathematics Subject Classification. Primary 58E10; Secondary 57R91, 53C22.

(C)1998 American Mathematical Society 
Notation and Definitions. By a Morse metric on $M$ we mean a metric with all closed geodesics nondegenerate (in the Morse theory sense). A curve $\sigma \in \Lambda$ has multiplicity $m$ if its stability group in $S O(2)$ is $\mathbb{Z}_{m}$. A curve of multiplicity 1 is prime. For $n \in \mathbb{Z}^{+}$, the $n^{\text {th }}$ power map is the map $\Lambda \rightarrow \Lambda$ by $\sigma \mapsto \sigma^{n}$, where $\sigma^{n}(t)=\sigma(n t)$. Let $\Lambda^{a} \subset \Lambda$ be the set of closed curves of energy $\leq a$. Let

$$
\begin{aligned}
& \omega_{n}=e^{2 \pi i / n} \text { generate } \mathbb{Z}_{n} \subset S O(2), \\
& T \text { generate } \mathbb{Z}_{2} \subset O(2), \\
& \delta_{n}=\left\{e^{2 \pi i t} \mid 0 \leq t \leq \frac{1}{n}\right\} \subset S O(2) .
\end{aligned}
$$

If $x$ is a $k$-chain on $\Lambda$, we use the prism operator [2] to form the $k+1$-chain $\delta_{n} x$. Thus $\delta_{n}$ is also a linear map on $C_{*}(\Lambda)$. Put

$$
\delta=\left(1+\omega_{n}+\cdots+\omega_{n}^{n-1}\right) \delta_{n} \quad("=S O(2) ") .
$$

Let $\mathbb{D}_{N} \subset O(2)$ be the dihedral group of order $2 N$, generated by $T$ and $\omega_{N}$.

Given a Morse metric on $M$, let

$$
\mathfrak{G}_{\lambda}\left(\text { resp. } \overline{\mathfrak{G}}_{\lambda}\right)=\{\text { closed geodesics of index } \lambda\} / S O(2)(\text { resp. } O(2)) \text {. }
$$

Let $\mathfrak{G}^{ \pm} \subseteq \mathfrak{G}, \overline{\mathfrak{G}}^{ \pm} \subseteq \overline{\mathfrak{G}}$ be the image of the set of closed geodesics for which the stability subgroup $\mathbb{Z}_{m}$ preserves $(+)$ or reverses (-) the orientation of the negative bundle.

Proposition I. Let $M$ be a compact surface with a Morse metric. Suppose all closed geodesics of energy $\leq a$ have multiplicity dividing $N$. Then $\Lambda^{a}$ has the equivariant homotopy type of a $C W$ complex $\mathcal{M}^{a}$ with cells

(i) lying on $\mathcal{M}^{0}=M=$ Fixed point set of $O(2)$,

(ii) $\omega_{N}^{k} D_{\sigma}, T \omega_{N}^{k} D_{\sigma}, 0 \leq k<\frac{N}{m}$ (summits),

(iii) $\omega_{N}^{k} \delta_{N} D_{\sigma}, T \omega_{N}^{k} \delta_{N} D_{\sigma}, 0 \leq k<\frac{N}{m}$ (tunnels),

with one $\mathbb{Z}_{m}$-cell $D_{\sigma}$ of dimension $\lambda$ for each $\sigma \in \overline{\mathfrak{G}}_{\lambda}$ with $E(\sigma) \leq a$, and $m=$ multiplicity $(\sigma)$. This $C W$ complex carries an $O(2)$-action which is $\mathbb{D}_{N}$-cellular. There are natural equivariant power maps $\mathcal{M}^{a} \rightarrow \mathcal{M}^{n^{2} a}$ and inclusions $\mathcal{M}^{a} \rightarrow \mathcal{M}^{b}$ if $a<b$ reflecting the power and inclusion maps on $\Lambda$.

Summit and tunnel are Klingenberg's terms for the unstable manifold (image in $\Lambda$ of the negative cell $D_{\sigma}$ ) of a closed geodesic $\sigma$ and its $S O(2)$-orbit. The first corollary says that it makes sense to work algebraically with the summits and tunnels and the boundary map $d$. Everything works equivariantly.

Corollary 1 (We can play "summits and tunnels"). Put

$$
\begin{aligned}
& \mathbb{M}_{k}=\mathbb{Z}\left[\mathbb{D}_{N}\right] \text {-module spanned by } \\
& \begin{cases}D_{\sigma}^{\cdot}, & \sigma \in \overline{\mathfrak{G}}_{k}, \\
\delta_{N} D_{\sigma}, & \sigma \in \overline{\mathfrak{G}}_{k-1},\end{cases}
\end{aligned}
$$

with relations, for $m=\operatorname{mult}(\sigma)$ and $\sigma \in \overline{\mathfrak{G}}^{ \pm}$,

$$
\left\{\begin{array}{l}
\left(1 \mp \omega_{m}\right) D_{\sigma}=0, \\
\left(1 \mp \omega_{m}\right) \delta_{N} D_{\sigma}=0 .
\end{array}\right.
$$


There is a boundary map $d: \mathbb{M}_{k} \rightarrow \mathbb{M}_{k-1}$ commuting with the $\mathbb{D}_{N}$-action;

$$
\begin{aligned}
& d^{2}=\delta_{N}^{2}=0, \\
& d \delta_{N}+\delta_{N} d=\left(1-\omega_{N}\right), \\
& \delta_{N} T+\omega_{N} T \delta_{N}=0, \\
& \delta_{N} \omega-\omega_{N} \delta_{N}=0 .
\end{aligned}
$$

$(\mathbb{M}, d)$ computes the homology of $\left(\Lambda^{a}, \Lambda^{0}\right)$. Moreover

$$
\operatorname{Ker} \delta=\operatorname{Image}\left(1-\omega_{N}\right)+\operatorname{Image}\left(\delta_{N}\right)+\operatorname{Span} \overline{\mathfrak{G}}^{-}
$$

and d descends to a map on $\mathbb{M} / \operatorname{Ker} \delta$, where

$$
\mathbb{M} / \operatorname{Ker} \delta \simeq \mathbb{Z}\left[\mathfrak{G}^{+}\right]
$$

Corollary 2. ("Going to the Morse complex"). Start with chains $x \in C_{k}\left(\Lambda^{a}\right), y \in$ $C_{k+\ell}\left(\Lambda^{b}\right)$ with

$$
\begin{aligned}
d x=0 & \text { and } & d y & =x(\ell=1) \\
& \text { or } & d y & =(1 \pm T) x(\ell=1) \\
& \text { or } & d y & =\delta x(\ell=2) .
\end{aligned}
$$

There exist (noncanonically) $\tilde{x} \in \mathbb{M}_{k}^{a}$ and $\tilde{y} \in \mathbb{M}_{k+\ell}^{b}$ satisfying the same equations and with the property that $x$ and $\tilde{x}$ have the same image in

$$
H_{k}\left(\Lambda^{a}\right) \cong \operatorname{Ker}\left(d: \mathbb{M}_{k}^{a} \rightarrow \mathbb{M}_{k-1}^{a}\right) / \operatorname{Im}\left(d: \mathbb{M}_{k+1}^{a} \rightarrow \mathbb{M}_{k}^{a}\right)
$$

Similarly, $y$ and $\tilde{y}$ have the same image in $H_{k+\ell}\left(\Lambda^{b}, \Lambda^{a}\right)$.

Corollary 3 ("Divisibility"). Let $\bar{x}, \bar{y} \in \mathbb{M} / \operatorname{Ker} \delta$ with $d \bar{y}=\bar{x}$. If $\bar{x} \neq 0$ then $\bar{y} \neq 0$; say

$$
\bar{x}=\sum_{\sigma} a(\sigma) \sigma \quad \text { and } \quad \bar{y}=\sum_{\tau} b(\tau) \tau
$$

with $a, b \in \mathbb{Z}$ and $\sigma, \tau \in \mathfrak{G}^{+}$(using the identification $(*)$ ); let $m(\sigma)=\operatorname{mult}(\sigma)$. Then the greatest common factor of the $b(\tau) m(\tau)$ divides each $a(\sigma) m(\sigma)$.

Conjecture. ("Approximating a degenerate geodesic by nondegenerate ones") Let $(M, g)$ be a compact manifold with only isolated closed geodesics. There is a smooth family of metrics $g_{t}, 0 \leq t \leq 1, g_{0}=g$, and, for each prime closed geodesic $\sigma$ on $(M, g)$, a finite number of continuous families $\tau_{0}, \tau_{1}, \ldots, \tau_{\ell(\sigma)}(\sigma, t)$ of closed curves on $M$ with $\tau_{i}(\sigma, t)$ a geodesic in the $g_{t}$ metric, $\tau_{i}(\sigma, t)$ prime for $t>0 ; \tau_{i}(\sigma, 0)=$ $\sigma^{m_{i}(\sigma)}$ for some $m_{i} \in \mathbb{Z}$, where $m_{0}=1$ and, if $\ell(\sigma) \geq 1$, for each $i>1$ the nullity of $\sigma^{m_{i}}$ exceeds the nullity of $\sigma^{n}$ for all proper divisors $n$ of $m_{i}$. Moreover, given $a>0$, there is an $\varepsilon>0$ so that

$g_{t}$ is a Morse metric on $\Lambda^{a}$ for $t \in(0, \varepsilon]$;

All geodesics of the $g_{t}$ metric with energy $\leq a$ are powers of some $\tau_{i}(\sigma, t)$;

If $E\left(\tau_{i}^{n}(\sigma, t)\right) \leq a$ and $t \in[0, \varepsilon]$ then

Index $\left(\tau_{i}^{n}(\sigma, t)\right)$ is constant in $t$ for $t>0$, Index $\left(\sigma^{m_{i}(\sigma) \cdot n}\right) \leq \operatorname{Index}\left(\tau_{i}^{n}(\sigma, t)\right) \leq \operatorname{Index}+\operatorname{Nullity}\left(\sigma^{m_{i}(\sigma) \cdot n}\right)$,

The conjugacy class of the Poincaré map of $\tau_{i}(\sigma, t)$ is continuous in $t$, For $|\rho|=1$ the $\rho$-index of $\tau_{i}(\sigma, t)$ is constant in $t$ away from the eigenvalues of the Poincaré map. 
We find the conjecture to be a useful way to image a degenerate closed geodesic. However for the applications we do not need the full conjecture, but only its topological consequences. The following proposition says that for a surface these consequences do hold; if all closed geodesics are isolated, the equivariant homotopy type of the nested spaces $\Lambda^{a}$ behaves as if the above conjecture were true. In particular, there is a nested set $\mathcal{M}^{a}$ of $C W$ complexes with $O(2)$-action and power maps carrying the $O(2)$-homotopy type of the spaces $\Lambda^{a}$ and with cells reflecting "phantom" prime closed geodesics $\tau_{i}(\sigma)$.

To state the proposition carefully we need to describe the contributions to the CW complex $\mathcal{M}^{a}$ coming from the iterates of a prime nondegenerate closed geodesic. To a closed geodesic $\tau$ on a surface are associated [1], [8] a length $L$, a Poincaré map $P \in S \ell(2, \mathbb{R})$ and a $\rho$-index $I:\{|\rho|=1\} \rightarrow \mathbb{Z}$. Bott [1] showed that $P$ determines $I$ up to an additive constant. The parity of this constant is determined by $P$ and by the orientability of the geodesic. We say a closed geodesic $\tau$ is orientable if the normal bundle of the immersion $\tau: S^{1} \rightarrow M$ is orientable. (This depends only upon the component of $\tau$ in $\Lambda$; on an orientable surface every closed geodesic is orientable.) From Klingenberg we have the following fact.

Lemma [9, pp. 291-292]. Let $\tau$ be a nondegenerate closed geodesic on a surface M. Assume $\tau$ is (resp. is not) orientable. If $\tau$ is elliptic, then $\operatorname{Index}(\tau)$ is odd (resp. even). If $\tau$ is hyperbolic, then $\operatorname{Index}(\tau)$ is even (resp. odd) if and only if the eigenvalues of $P$ are positive.

Here $\operatorname{Index}(\tau)=I(1)$; on a surface the eigenvalues of $P$ have product 1 and are real or complex conjugates; elliptic means they are not real, and hyperbolic that they are real but not \pm 1 .

If $\tau$ is a closed geodesic, $L, P$, and $I$ determine the length, index and nullity of all iterates of $\tau$. Bott proved

$$
\begin{aligned}
\operatorname{Index}\left(\tau^{n}\right) & =\sum_{\rho^{n}=1} I(\rho) . \\
\operatorname{Nullity}\left(\tau^{n}\right) & =\sum_{\rho^{n}=1} \operatorname{dim} \operatorname{Ker}(P-\rho I) .
\end{aligned}
$$

(The geodesic $\tau^{n}$ is nondegenerate if and only if $\operatorname{Nullity}\left(\tau^{n}\right)=0$.) They also determine the assignment of $\tau^{n}$ to $\mathfrak{G}^{+}$or $\mathfrak{G}^{-}$according to [8, p. 128].

$$
\tau^{n} \in \mathfrak{G}^{-} \Leftrightarrow n \text { is even and } \operatorname{Index}\left(\tau^{2}\right)-\operatorname{Index}(\tau) \text { is odd. }
$$

Thus for a Morse metric the $\mathbb{Z}\left[\delta, \mathbb{D}_{N}\right]$-modules $\mathbb{M}_{k}^{a}$ are determined by the data $(L, P, I)$ for each prime closed geodesic.

Proposition II. Let $M$ be a compact surface with only isolated closed geodesics. To each prime closed geodesic $\sigma$ one can assign a finite number of continuous families

$$
(L, P, I)(\sigma, i, t), \quad 0 \leq i \leq \ell(\sigma) .
$$

Thus for each $\sigma, i$ and $t \in[0,1]$ we have a length $L \in \mathbb{R}$, a matrix $P \in S \ell(2, \mathbb{R})$ and a $\rho$-index $I:\{|\rho|=1\} \rightarrow \mathbb{Z}$, which we imagine as belonging to the phantom prime closed geodesic $\tau_{i}(\sigma, t)$. If all iterates of $\sigma$ are nondegenerate, then $\ell(\sigma)=0 . L, P$, and $I$ are continuous in $t$; when $t=0$ they should equal the length, Poincaré map and $\rho$-index of the honest geodesic $\sigma^{m_{i}}$. (Here $m_{0}=1$; for a surface, if $\ell(\sigma) \geq 1$, then $m_{i}(\sigma)$ is the minimum iterate of $\sigma$ which is degenerate for each $i>1$.) $I$ 
and $P$ are related in the way described by Bott; moreover the parity of $I$ is to be as specified in the lemma above (or in its generalization below). (In order to decide whether $\tau_{i}$ is orientable we assume that $\tau_{i}(\sigma, t)$ lies in the same component of $\Lambda$ as $\sigma^{m_{i}}$.) Let the index and nullity of $\tau_{i}^{n}$ be defined by (1), (2), $P$ and I. Given $a>0$, there is an $\varepsilon>0$ so that for $0<t<\varepsilon$ and $n L(\sigma, i, t)<a$,

$$
\operatorname{Nullity}\left(\tau_{i}^{n}(\sigma, t)\right)=0
$$

(i.e. the phantom metric is "Morse" on $\left.\Lambda^{a}\right)$. Moreover,

$$
\begin{gathered}
\operatorname{Index}\left(\tau_{i}^{n}(\sigma, t)\right) \quad \text { is constant in } t \\
\operatorname{Index}\left(\sigma^{m_{i}(\sigma) \cdot n}\right) \leq \operatorname{Index}\left(\tau_{i}^{n}(\sigma, t)\right) \leq \operatorname{Index}+\operatorname{Nullity}\left(\sigma^{m_{i}(\sigma) \cdot n}\right) .
\end{gathered}
$$

For each $t \in(0, \varepsilon), \Lambda^{a}$ has the homotopy type of a complex as in Proposition I, with one $\mathbb{Z}_{n}$-cell of dimension $\lambda$ for each $(n, \sigma, i, t)$ with $n L(\sigma, i, t)<a$ (think: for each phantom $\tau_{i}^{n}(\sigma, t)$ with length $\left.<a\right)$ and index $\left(\tau_{i}^{n}(\sigma, t)\right)=\lambda$. The action of $\mathbb{Z}_{n}$ on the cell is consistent with (3).

In the course of the proof of Proposition II we will prove a generalization of the lemma quoted above to the effect that, for any closed geodesic, the parity of $I$ is determined by the conjugacy class of $P$ and the orientability of the geodesic. The proof is simple and also works in higher dimensions. An explicit description of the index $I(1)$ in terms of the matrix $P$ would be complicated, but the first figure below should illuminate both how to find the parity of $I$ in terms of $P$, and the idea of the proof.

Remarks. The propositions are easy to prove, and both follow from a special property of the free loop space: While the $S O(2)$-action is not free, the fixed point set of $\mathbb{Z}_{n}$ is the image under the power map of a set where the action is "more" free. To prove the first proposition, we need to equivariantly attach a cell on which $\mathbb{Z}_{n}$ acts. For a surface, the formula for the index of the iterates [1] ensures that if we do this on the fixed point sets of $\mathbb{Z}_{m}, m \mid n$, by the power map, we will be attaching to the appropriate sketeton. On the rest of the cell, $\mathbb{Z}_{n}$ acts freely and presents no difficulty. The proof of the second proposition follows from the proof of the theorem of Gromoll and Meyer [3]: Since the characteristic manifold is equivariant and "iterates" by the power map, it is enough to equivariantly resolve the energy function on the characteristic manifold to a Morse function the first time that it appears.

If $M$ has a Morse metric, "ordinary" Morse theory gives as a model for $\Lambda M$ a $C W$ complex with one $\lambda$-cell $D_{\sigma}$ (summit) and one $(\lambda+1)$-cell $S^{1} \times D_{\sigma} / \mathbb{Z}_{m}$ (tunnel) for each oriented closed geodesic $\sigma$ of index $\lambda$ and multiplicity $m$. In general it is not clear how to construct this $C W$ complex equivariantly. (See the very end of this paper.) Thus the model $C W$ complex carries the homotopy type, but not the $O(2)$-homotopy type, of $\Lambda$. The importance of the group action was recognized by Morse. Rademacher [12] has constructed a "bigger" equivariant morse complex than that in Proposition I using the strata of the $\mathbb{Z}_{m}$-action on $D_{\sigma}$ as cells. Our complex uses only the $\mathbb{D}_{N}$ orbits of the cells $D_{\sigma}, \delta_{N} D_{\sigma}$ and no lower dimensional strata. Thus for surfaces we have found an equivariant version of Morse's original complex. In [5] we make some comment as to why smaller may be better. Note, however, that the power maps are cellular in Rademacher's complex but not in ours. Moreover, we use his complex in an essential way to prove the existence of ours. Our idea is not that this complex should "replace" Rademacher's complex; 
most useful should be the "combined" complex obtained by altering the attaching maps in Rademacher's complex as in the proof of Proposition I. This yields an $O(2)$-topological space $\mathcal{M}^{a}$ with, simultaneously and coherently, the structure of a $\left(\mathbb{Z}_{N}, O(2)\right)$-CW complex with cellular power maps and that of an ordinary $\mathrm{CW}$ complex with an $O(2)$-action as in Proposition I.

In Corollary $1, \mathbb{M}$ is a module spanned over the ring $\mathbb{Z}\left[\mathbb{D}_{N}, \delta_{N}\right]$ by the $D_{\sigma}, \sigma \in \overline{\mathfrak{G}}$. In general if $X$ is an $O(2)$-space, any subcomplex $\mathbb{M}$ of $C_{*}(X)$ that computes $H_{*}(X)$ and that is also a module over $\mathbb{Z}\left[D_{N}, \delta_{N}\right]$ also computes the equivariant homology of $X$ for the groups $O(2), S O(2)$, and subgroups of $\mathbb{D}_{N}$. (This is a stronger version of the useful but somewhat disappointing fact that an equivariant map which is a homology equivalence induces an isomorphism in equivariant homology.) For example $\mathbb{D}_{N}$ or $O(2)$-homology is represented in a triple complex with entries in $\mathbb{M}$ and maps given by $d, \delta_{N}, \mathbb{D}_{N}$. The various group inclusions induce natural maps on the complexes computing these homologies. We hope to describe this in a later paper. (For the complex giving $\mathbb{D}_{N}$ homology, see [10]. For $S O(2)$-homology, see $[4,7]$.) The fact that we get such a complex when $X=\Lambda$ using one generator $D_{\sigma}$ for each closed geodesic $\sigma \in \overline{\mathfrak{G}}$ represents, we think, an ideal relationship between geometry and topology.

The "trick" of Corollary 2 can be repeated for a string of chains $x_{1}, \ldots, x_{n}$ with

$$
d x_{1}=0 \text { and } d x_{i}=\left(1+(-1)^{i} T\right) x_{i-1} \text { or } d x_{i}=\delta x_{i-1} .
$$

These correspond to strings of subordinated classes where we are taking cap products in the first case with the basic class $\omega_{1} \in H_{1}(B O(2))$ and in the second case with $\omega_{2} \in H_{2}(B O(2))$. All this has a natural expression in terms of equivariant homology. Moreover, Corollary 2 also works with equivariant chains $x, y \in C_{*}^{O(2)}(\Lambda)$ and $d y=x$. The author hopes to include the details in the as yet unwritten paper mentioned in the previous paragraph.

Corollary 3 should be compared to the Klingenberg divisibility lemma [8, pp. $147-150$ ], which states essentially that, in a special case, $b(\tau) m(\tau)$ divides $a(\sigma) m(\sigma)$. We see no reason why this should be true in general; however, in the case of a surface the corollary as stated is still useful due to Corollary 4 below.

In Corollary 3 we have in mind that $\bar{x}, \bar{y}$ are the images of $\tilde{x}, \tilde{y}$ from Corollary 2 . The condition $\bar{x} \neq 0$ will then be satisfied if the image of $x$ in $H_{*}\left(\Lambda^{a} / S O(2), \Lambda^{0} ; \mathbb{Q}\right)$ is nonzero. (See the last remark below.)

Proposition II gives a "limiting" Morse complex for $\Lambda(M, g)$ having all the proper-ties stated in Proposition I. The iterates of the limiting "phantom" prime closed geodesics $\tau_{i}(\sigma)$ have

$$
\operatorname{Index}\left(\tau_{i}^{m}(\sigma)\right)=\lim _{t \rightarrow 0} \operatorname{Index}\left(\tau_{i}^{m}(\sigma, t)\right) .
$$

We attach $\mathbb{Z}_{m}$-cells as in Proposition I for these iterates. (If the conjecture were true, for fixed $a$ the truncated Morse complex $\mathcal{M}^{a}$ for $(M, g)$ would be the "same" (same cells, homotopic attaching maps) as the truncated Morse complex coming from $g_{t}, t<\varepsilon$.) (Note that if $(M, g)$ has finitely many closed geodesics $\sigma$ there will be finitely many $\tau_{i}(\sigma)$.) This Morse complex will differ from the Morse complex coming from a Morse metric only in that

1) The sequence of indices of the iterates of $\tau_{i}(\sigma)$ will not, in general, look like that coming from a nondegenerate closed geodesic, but will be a limit of such sequences. (See the proof below for an example.) 
2) Associated to each cell $D_{\sigma}$ of $\mathcal{M}$ is the length $L(\sigma)$.

For a Morse metric each $D_{\sigma}$ is attached to cells of lesser length. In the isolated degenerate case we will need either to allow attaching to cells of equal length (putting length $\left(\tau_{i}(\sigma)\right)=$ length $\left(\sigma^{m_{i}}\right)$ ), or to allow the length of $\tau_{i}(\sigma)$ in $\mathcal{M}^{a}$ to vary with $a$. (See the proof below.)

Corollary 4. Let $(M, g)$ be a compact orientable surface with only isolated closed geodesics; $\mathcal{M}, \mathbb{M}$ as above. Let $p>2$ be prime. Then $\mathbb{M}_{2 p} / \operatorname{Ker} \delta$ is spanned by $\tau$ with $\tau \in \mathfrak{G}^{+}$and with $\tau$ prime or with $p=\operatorname{mult}(\tau)$.

In the nondegenerate case Corollary 4 follows from the lemma above; in the degenerate case we need the parity of the $\rho$-index $I$ to be "appropriate." In the degenerate case Corollary 4 seems remote from geometry, as the spanning geodesics $\tau$ are only limiting phantoms; however, note that Corollary 4 "feeds into" Corollary 3 if there are no "prime closed geodesics" $\tau_{i}(\sigma)$ with index $2 p$.

The "circular connectivities" computed by Morse [11, p. 349] are the mod 2 Betti numbers of the complex

$$
\mathbb{M} / \operatorname{Ker} \delta(1+T) \simeq \mathbb{Z}\left[\overline{\mathfrak{G}}^{+}\right]
$$

for the "limiting ellipsoid" metric on the sphere. It seems that Morse was trying to compute the mod 2 Betti numbers of the quotient $\Lambda / O(2)$; it is well known that Morse's circular connectivities are not a topological invariant. The complex $\mathbb{M} / \operatorname{Ker} \delta$ (or $\mathbb{M} / \operatorname{Ker} \delta(1+T)$ ) is geometrically appealing since it has one generator over $\mathbb{Z}$ for each closed geodesic in $\mathfrak{G}^{+}\left(\right.$resp. $\left.\overline{\mathfrak{G}}^{+}\right)$. If we take rational coefficients, the homology of the quotient space $\left(\Lambda / S O(2), \Lambda^{0}\right)$ is computed by $H_{*}(\mathbb{M} / \operatorname{Ker} \delta)$. (This can be proved using results in [6].) The latter is not a topological invariant if we take integer coefficients. (If you try to prove that it is, you will see that the problem is that $\operatorname{Ker}\left(1-\omega_{N}\right) \neq \operatorname{Im}\left(1+\omega_{N}+\cdots+\omega_{N}^{N-1}\right)$ over $\mathbb{Z}$. $)$ As an example take a $\mathbb{Z}_{k}$-disk $D$ of dimension 2 and a function $f$ with a nondegenerate maximum at the origin; then perturb $f$ as in the proof of Proposition II to get a new function $g$. One expects $g$ to have for example a minimum $\tau_{0}$ at the origin, $k$ saddle points $\left\{\omega_{k}^{i} \tau_{1}\right\}$ and $k$ maxima $\left\{\omega_{k}^{i} \tau_{2}\right\}$ with boundaries $(\bmod \partial D)$

$$
\begin{aligned}
d D_{\tau_{2}} & =\left(1-\omega_{k}\right) D_{\tau_{1}}, \\
d D_{\tau_{1}} & =D_{\tau_{0}} .
\end{aligned}
$$

(The graph of $g$ would be an appropriate saddle for a $k$-legged spider with a protruding abdomen.) Now extend $f, g$ equivariantly to $D \times_{\mathbb{Z}_{k}} S^{1}$. We have

$$
H_{n}\left(\mathbb{M}_{g} / \operatorname{Ker} \delta\right)=H_{n}\left(\mathbb{M}_{f} / \operatorname{Ker} \delta\right)= \begin{cases}\mathbb{Z} & \text { if } n=2, \\ 0 & \text { otherwise }\end{cases}
$$

but $H_{2}\left(\mathbb{M}_{f} / \operatorname{Ker} \delta\right) \rightarrow H_{2}\left(\mathbb{M}_{g} / \operatorname{Ker} \delta\right)$ is multiplication by $k$; there is no map in the other direction over $\mathbb{Z}$. (Note that Corollary 3 and Corollary 4 fit in well with the above example.)

Proof of Proposition I. The property of surfaces we use is the following: For every pair $\sigma, \tau$ of closed geodesics in the same component of $\Lambda$ we have, for $i=0$ and $i=1$,

$$
\text { index }(\tau)<\operatorname{index}(\sigma)-i \Longrightarrow \operatorname{index}\left(\tau^{m}\right)<\operatorname{index}\left(\sigma^{m}\right)-i \quad \forall m .
$$


This follows from the lemma mentioned above, the fact $[8, \mathrm{p} .97]$ that $\operatorname{Index}\left(\tau^{m}\right)=$ $m \cdot \operatorname{Index}(\tau)$ for a hyperbolic closed geodesic, and the fact $[8$, p. 98] that

$$
m \cdot \operatorname{Index}(\tau)-m+1 \leq \operatorname{Index}\left(\tau^{m}\right) \leq m \cdot \operatorname{Index}(\tau)+m-1
$$

for any closed geodesic $\tau$ on a surface.

Our starting point is a theorem of Rademacher [12]; the reader is referred to that paper for definitions.

Theorem (Rademacher). Let $M$ be a compact Riemannian manifold with a Morse metric and $\left(a_{i}\right)_{i \geq 1}$ a sequence of regular values of $E$ with $a_{i}<c_{i}<a_{i+1}$ and $\left(c_{i}\right)$ the strictly increasing sequence of critical values of $E$. Then there is a $G$ - $C W$ complex $X\left(G=S^{1}, O(2)\right)$ with subcomplex $A=\Lambda^{0} M G$-homotopy equivalent to $\Lambda M$. Moreover there is a sequence $\left(m_{i}\right)_{i \geq 1} \subset \mathbb{N}$ such that $\left.m_{i}\right|_{i+1}$ and a filtration $\left(X_{i}\right)_{i \geq 1}$ of $X$ by closed $G$-subspaces such that $\left(X_{i}, A\right)$ is a relative $\left(\mathbb{Z}_{m_{i}}, G\right)$-CW complex which is with its canonical $\left(\mathbb{Z}_{m_{i}+1}, G\right)$-CW structure a subcomplex of $\left(X_{i+1}, A\right) . X_{i}$ is G-homotopy equivalent to $\Lambda^{a_{i}} M$.

The topological space $X$ is formed inductively by attaching the cells $D_{\sigma}=D^{\lambda}$ and $\delta_{m} D_{\sigma}$ for a closed geodesic $\sigma$ of index $\lambda$ and multiplicity $m$. The cells of the complex $X$ are strata of the $\mathbb{Z}_{m}$ action on the cells $D_{\sigma}$.

Starting with the geodesics of smallest energy, we alter the attaching map

$$
f_{\sigma}: S^{\lambda-1} \rightarrow X, \quad S^{\lambda-1}=\partial D_{\sigma},
$$

so that the image lies in

$$
X_{\sigma}^{-}=A \cup \bigcup_{\substack{\lambda(\tau) \leq \lambda-1 \\ E(\tau)<E(\sigma)}} \omega_{N}^{k} T^{\ell} D_{\tau} \cup \bigcup_{\substack{\lambda(\tau)<\lambda-1 \\ E(\tau)<E(\sigma)}} \omega_{N}^{k} T^{\ell} \delta_{n} D_{\tau} .
$$

The result will be a $\left(\mathbb{Z}_{N}, O(2)\right)$-CW complex which has all the stated properties of $X$, but which also carries the structure of an ordinary CWcomplex $\mathcal{M}$ with $O(2)$-action and with only top-dimensional cells, as described in Proposition I.

Now suppose we have done this for all closed geodesics with energy less than $E(\sigma)$. Then the right hand side above will be a subcomplex of $X$. If $\sigma$ has multiplicity $m, S^{\lambda-1}$ has the structure of a $\mathbb{Z}_{m}$-complex [12]. We first attach the fixed point sets of $\mathbb{Z}_{n}$ in $S^{\lambda-1}, n \mid m$, by the power maps. (Rademacher does not explicitly mention the power maps; however, it is clear that the complex $X$ can be constructed respecting the power maps $D_{\tau} \rightarrow D_{\tau^{n}}$.) Note that the union $F$ of the fixed point sets of $\mathbb{Z}_{n}$ is a sub- $\mathbb{Z}_{m}$-complex of $S^{\lambda-1}$, and that these sets are attached cellularly in $X$. The hypothesis on the growth of the index implies that $F$ is attached to $X_{\sigma}^{-}$. Let $Y^{j}$ be the $j$-skeleton of $S^{\lambda-1}$; assume that $F \cup Y^{j-1}$ has been attached with $Y^{j-1}$ attached along the $j-1$-skeleton of $X_{\sigma}^{-}$. Let $e=D^{j} \times \mathbb{Z}_{m}$ be a $j$-cell of $S^{\lambda-1}$; note that $\mathbb{Z}_{m}$ acts freely on the remaining strata. We start with an attaching map

$$
f: D^{j} \longrightarrow \bigcup_{E(\tau)<E(\sigma)} \delta D_{\tau} .
$$

Suppose the image of $f$ meets the interior of some cell in some $\delta D_{\tau}$ which is not in $X_{\sigma}^{-}$. Assume $E(\tau)$ is maximal. By induction, there is an ordinary CW complex with the following cells:

1. All cells of $X$ with energy less than $E(\tau)$;

2. All cells of $X$ lying on $X_{\sigma}^{-}$; 
3. Cells $D_{\mu}$ and $\delta_{N} D_{\mu}$ with $E(\mu)=E(\tau)$.

Note that in 1 and 2 we include all the cells of $X$, but in 3 we include only top-dimensional cells. The image of the attaching map $f$ lies in the above CW complex; thus, keeping $f$ fixed on $Y^{j-1}$, we can assume that the image of $f$ lies in the $j$-skeleton of the above complex and thus "misses" $D_{\mu}$ if $\lambda(\mu) \geq \lambda$ and $\delta D_{\mu}$ if $\lambda(\mu) \geq \lambda-1$. We extend $f$ to the rest of $e$ using the $\mathbb{Z}_{m}$-action.

Proof of the Corollaries. Corollary 1 follows immediately, since

$$
\mathbb{M}_{k} \cong H_{k}\left(\mathcal{M}^{k}, \mathcal{M}^{k-1}\right),
$$

as $\mathbb{Z}\left[\mathbb{D}_{N}\right]$-modules. It is straightforward to check that the relations involving $\delta_{N}$ and $d$ hold on the singular chain complex and thus on $\mathbb{M}_{k}$.

To prove Corollary 2, we push $x$ and $y$ forward to $\mathcal{M}$ using homotopy equivalence, then retract $x$ to $\mathcal{M}^{k}$ and $y$ to $\mathcal{M}^{k+\ell}$. Then take the image in $\mathbb{M}$ by the above equivalence.

Proof of Corollary 3. If $N, p$, and $q$ are natural numbers with $N=p q$, let

$$
S(N, p)=1+\omega_{N}+\omega_{N}^{2}+\cdots+\omega_{N}^{q-1} .
$$

Let

$$
F: \mathbb{Z}\left[\mathbb{Z}_{N}\right] \rightarrow \mathbb{Z} \quad \text { by } \omega_{N} \mapsto 1 .
$$

Then:

(i) $\left(1-\omega_{N}\right) S(N, p)=\left(1-\omega_{p}\right)$ in $\mathbb{Z}\left[\mathbb{Z}_{N}\right]$.

(ii) $F S(N, p)=q$.

(iii) $\operatorname{Ker}\left(1-\omega_{N}\right)=\operatorname{Image} S(N, 1)$.

If $X=\sum_{\sigma} A(\sigma) D_{\sigma} \in \mathbb{M}$ with $A \in \mathbb{Z}\left[\mathbb{Z}_{N}\right]$, then the image of $X$ in $\mathbb{M} / \operatorname{Ker} \delta$ is $\sum_{\sigma} F A(\sigma) \sigma$.

Let $n$ be a fixed natural number. If $n$ divides $b m$ for each $\tau$, we can find a preimage $Y \in \mathbb{M}$ for $\bar{y}$ with $\left(1-\omega_{n}\right) Y=0$ : Suppose $n=t(\tau) r(\tau)$ with $t$ dividing $b$ and $r$ dividing $m$. Let $D_{\tau} \in \mathbb{M}$ lie above $\tau$, and put

$$
Y=\sum_{\tau} \frac{b}{t} S(n, r) D_{\tau}
$$

If $X=d Y$, then also $\left(1-\omega_{n}\right) X=0$. Now

$$
X=\sum A(\sigma) D_{\sigma}
$$

with $A \in \mathbb{Z}\left[\mathbb{Z}_{N}\right]$ and $F(A)=a$. Since $\left(1-\omega_{n}\right) X=0$, for each $\sigma$ we have

$$
\left(1-\omega_{n}\right) A=\left(1-\omega_{m}\right) B
$$

for some $B \in \mathbb{Z}\left[\mathbb{Z}_{N}\right]$. (We have suppressed the dependence of $A, m$, and $B$ upon $\sigma$.) By (i), for each $\sigma$ this becomes

$$
\left(1-\omega_{N}\right) S(N, n) A=\left(1-\omega_{N}\right) S(N, m) B,
$$

which implies by (iii)

$$
S(N, n) A=S(N, m) B+S(N, 1) C
$$

for some $C \in \mathbb{Z}\left[\mathbb{Z}_{N}\right]$. Applying $F$ and using (ii), we have

$$
m a=b n+n m c,
$$

and thus $n$ divides each $m a$. 
Proof of Proposition II. If $\sigma$ is a prime closed geodesic on a surface, there is an integer $k$ so that

$$
\operatorname{Nullity}\left(\sigma^{n}\right)= \begin{cases}0 & \text { if } k \nmid n, \\ \operatorname{Nullity}\left(\sigma^{k}\right) & \text { if } k \mid n .\end{cases}
$$

Moreover, the characteristic manifold $[3,8]$ of $\sigma^{k}$ iterates by the power map to give a characteristic manifold for $\sigma^{n}$ if $k \mid n$. If $J=\operatorname{Nullity}\left(\sigma^{k}\right)$ then $J \leq 2$; we assume $J>0$. The characteristic manifold is then a $J$-disk $D$ with a $\mathbb{Z}_{k}$-action which is free except at the origin and whose derivative there is a rotation, of order $k$. Let $m_{i}=k$ for $i>0$.

We begin by "pushing down the center" by adding a function $\rho(x)|x|^{2}$ to the restriction of the energy function to $D$, where $\rho$ is a $\mathbb{Z}_{k}$-invariant bump function with small support and small absolute value which is a positive constant near the origin. This makes the origin a nondegenerate minimum. A small perturbation of the function with small support, but with support away from the origin, will now result in a $\mathbb{Z}_{k}$-invariant Morse function on the disk. We let $\tau_{0}(\sigma)=\sigma$ and let $\tau_{1}, \ldots, \tau_{\ell}$ be representatives of the $\mathbb{Z}_{k}$ orbits of the additional critical points of the perturbed function. If $D^{-}$is the subset of $D$ where the original energy function is less than $E(\sigma)$, then $\left(D^{-} \cup \sigma^{k}, D^{-}\right)$now has the equivariant homotopy type of a relative $C W$ complex with an $O$-cell $\tau_{0}^{k}$ fixed by $\mathbb{Z}_{k}$ and cells $\omega_{k}^{j} E_{\tau_{i}}$ of dimension $\leq J$ for each $\tau_{i}, i \geq 1$.

Now let $D_{\sigma^{n}}=D_{\tau_{0}^{n}}$ be the negative bundle of $\sigma^{n}$. The disk $D_{\sigma^{n}}$ carries a $\mathbb{Z}_{n}$-action fixing the origin. The negative-plus-null bundle of $\sigma^{n k}$ is

$$
D_{\sigma^{n k}} \times D^{n} \approx D_{\sigma^{n k}} \times D .
$$

Put

$$
D_{\tau_{i}^{n}}=D_{\sigma^{n k}} \times E_{\tau_{i}} \subseteq D_{\sigma^{n k}} \times D, \quad i \geq 1 .
$$

The group $\mathbb{Z}_{n k}$ acts on the $D_{\tau_{i}^{n}}$ by

$$
\omega_{k n}(x, y)=\left(\omega_{k n} x, \omega_{k} y\right) \text {. }
$$

Thus $\mathbb{Z}_{n}$ takes each $D_{\tau_{i}^{n}}$ to itself. Suppose for simplicity that $\sigma^{n k}$ is the only critical point with energy $a \in \mathbb{R}$. The iteration of the characteristic manifold $[3,8]$ implies that $\left(\Lambda^{a+\varepsilon}, \Lambda^{a-\varepsilon}\right)$ has the equivariant homotopy type of a relative $C W$ complex (with $O(2)$-action) with the cells

$$
\begin{aligned}
& D_{\sigma^{n k}}, T D_{\sigma^{n k}}, \\
& \delta_{n k} D_{\sigma^{n k}}, T \delta_{n k} D_{\sigma^{n k}}, \\
& \omega_{n k}^{j} D_{\tau_{i}^{n}}, T \omega_{n k}^{j} D_{\tau_{i}^{n}}, 0 \leq j<k, \\
& \omega_{n k}^{j} \delta_{n k} D_{\tau_{i}^{n}}, T \omega_{n k}^{j} \delta_{n k} D_{\tau_{i}^{n}}, 0 \leq j<k .
\end{aligned}
$$

These cells can be attached equivariantly by induction as in the proof of Proposition I (or as in [12]) by starting with the fixed point sets. Thus the tower $\left\{\sigma^{n}\right\}$ looks up to homotopy like the towers $\left\{\tau_{i}^{n}\right\}$. These "phantom" geodesics have index

$$
\begin{gathered}
\operatorname{Index}\left(\tau_{0}^{n}\right)=\operatorname{Index}\left(\sigma^{n}\right), \\
\operatorname{Index}\left(\tau_{i}^{n}\right)=\operatorname{Index}\left(\sigma^{n k}\right)+\operatorname{dim} E_{\tau_{i}}, \quad i \geq 1 .
\end{gathered}
$$

For $i \geq 1$, the $\mathbb{Z}_{n}$ action preserves the orientation of $D_{\tau_{i}^{n}}$ if and only if it preserves the orientation of $D_{\sigma^{n k}}$. Since $\operatorname{Index}\left(\sigma^{2 k}\right)-\operatorname{Index}\left(\sigma^{k}\right)$ is the dimension of the 

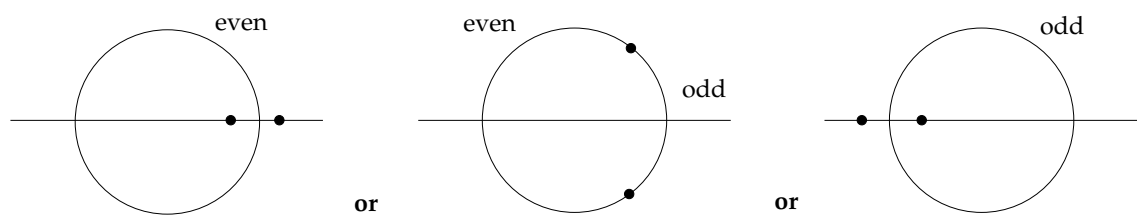

Figure 1

subspace of $D_{\sigma^{2 k}}$ on which $\mathbb{Z}_{2}$ acts as -1 [8, p. 128], the $\tau_{i}^{n}$ will be appropriately assigned to $\mathfrak{G}^{-}$or $\mathfrak{G}^{+}$.

It remains to be seen that, for any $N$, the index of $\left\{\tau_{i}^{n}\right\}_{i \leq n \leq N}$ can be achieved by an "appropriate" Poincaré map $P$ and $\rho$-index $I$. The lemma mentioned before Proposition II is the nondegenerate case of the

General Lemma. The conjugacy class of the Poincaré map of a closed geodesic and the orientability of the geodesic determine the parity of the $\rho$-index.

More special cases can be found in [9, p. 288-292]; we give here a simple proof covering all cases.

Proof of the general lemma. If $\sigma$ is an orientable (resp. non-orientable) closed geodesic on a surface and $\lambda \in \mathbb{R}$, then we have the differential equation

$$
\frac{D^{2} X}{d t^{2}}+R(\dot{\sigma}, X) \dot{\sigma}=-\lambda X
$$

subject to periodic (respectively anti-periodic) boundary conditions. Associated to the differential equation are a symplectic matrix $P_{\lambda} \in G=S \ell(2, \mathbb{R})$ and a $\rho$-index $I_{\lambda}$, a map from the unit circle $|\rho|=1$ to the whole numbers. (When $\lambda=0$ the equation is the Jacobi equation, $P_{\lambda}$ is the Poincaré map and $I_{\lambda}$ the $\rho$-index of $\sigma$; in general $P_{\lambda}$ is the transition matrix of the space of " $\lambda$-Jacobi-fields" along $\sigma$ and e.g. $I_{\lambda}(1)$ is the number of periodic $\mu$-jacobi fields along $\sigma$ with $\mu<\lambda$.) Bott showed [1] that the conjugacy class of $P_{\lambda}$ determines $I_{\lambda}$ up to a constant. In particular the eigenvalues of $P_{\lambda}$ are either $e^{ \pm i \theta}$ or $r$ and $\frac{1}{r}, r \in \mathbb{R} ; I_{\lambda}$ is constant away from the eigenvalues of $P_{\lambda}$, and the jump of $I_{\lambda}$ at a simple eigenvalue of $P_{\lambda}$ is always \pm 1 . The map $\lambda \mapsto\left(P_{\lambda}, I_{\lambda}\right)$ is continuous in the sense $I_{\lambda+\varepsilon}=I_{\lambda}$ away from the eigenvalues of $P_{\lambda}$ and where we take the usual topology on $G$. Now for $\lambda \ll 0, I_{\lambda} \equiv 0$ and the eigenvalues of $P_{\lambda}$ are positive and real (resp. negative and real). Continuity then implies in the orientable case that if $P_{\lambda}$ has positive real eigenvalues then $I_{\lambda}$ is even (except possibly at 1); if $P_{\lambda}$ has no real eigenvalues then $I_{\lambda}(-1)$ is even and $I_{\lambda}(1)$ is odd; if $P_{\lambda}$ has negative real eigenvalues then $I_{\lambda}$ is odd (except possibly at -1 ). Figure 1 shows the eigenvalues and the parity of $I$ in $\mathbb{C}$.

If $\sigma$ is not orientable, switch all parities. Because the $\rho$-index is determined by the conjugacy class of $P$ and by its value at any one point $\rho$, this proves the general lemma. (The same argument restricts the parity of $I$ for a closed geodesic on a manifold of higher dimension, a fact of which we are unaware in the literature.) Topologically $G$ is a solid torus; $\{(P, I) \mid I$ associated to $P\}$ is half (because we assume $I \geq 0$ ) of two copies of the universal cover of $G$, like two intertwined spiral staircases.

Assume now that $P_{0}$ is "degenerate", i.e. that if $P_{0}$ were the Poincaré map of a closed geodesic, that geodesic would be degenerate. This means that $(P-1)^{2}=0$ 


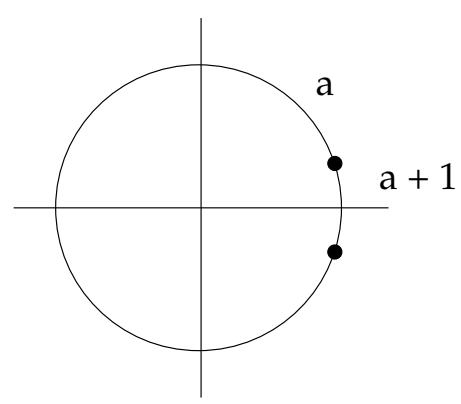

Figure 2

[1]. Let $I_{0}$ be associated to $P_{0}$. Given an integer $j$ with $0 \leq j \leq \operatorname{Nullity}\left(P_{0}-1\right)$, there is a path $\left(P_{t}, I_{t}\right)$ in the covering space starting at $\left(P_{0}, I_{0}\right)$ with $P_{t}$ nondegenerate and $I_{t}(1)=I_{0}(1)+j$ for $t>0$. (This is true for general reasons [1]; in any case the topology of $G$ is well-known and this is easy to check in each of the three possible conjugacy classes for $P_{0}$, given the splitting numbers [8, p. 99].)

Now, given $\sigma, \tau_{i}$ as above with $i \geq 1$, let $P_{0}$ and $I_{0}$ be the Poincaré map and $\rho$-index of $\sigma^{k}$, and let $j=\operatorname{dim}\left(E_{\tau_{i}}\right)$; then $j \leq J=\operatorname{Nullity}\left(P_{0}-I\right)$. If we assign to $\tau_{i}(\sigma, t)$ the Poincaré map $P_{t}$ and $\rho$-index $I_{t}$ given in the previous paragraph, it follows from the index formula [1],

$$
\operatorname{Index}\left(\tau_{i}^{n}\right)=\sum_{\rho^{n}=1} I_{t}(\rho),
$$

that the index of $\tau_{i}^{n}, 1 \leq n \leq N$, will be $\operatorname{Index}\left(\sigma^{n k}\right)+j$ for $t$ sufficiently small (depending on $N$ ), as desired. Under these circumstances $\tau_{i}^{n}$ will also be nondegenerate for $1 \leq n \leq N$, since $\tau_{i}^{n}$ is degenerate if and only if $P_{t}^{n}$ has 1 as an eigenvalue. The $\rho$-index $I_{t}$ will have the appropriate parity, since the path started at a point $\left(P_{0}, I_{0}\right)$ in the correct component of the covering space $\{(P, I)\}$, depending on the orientability of $\sigma^{k}$. A similar argument produces $P_{t}$ and $I_{t}$ when $i=0$.

As an example, suppose that $M$ is orientable and that $\operatorname{dim} E_{\tau_{i}}=2$. Then the Poincaré map $P_{\sigma^{k}}$ of $\sigma^{k}$ is the identity and there is an even integer $a$ so that

$$
\operatorname{Index}\left(\sigma^{n k}\right)=a n-1 \text {, }
$$

so

$$
\operatorname{Index}\left(\tau_{i}^{n}\right)=a n+1 \text {. }
$$

(The first equality follows from the fact that $\left(P_{0}, I_{0}\right)$ must be close to nondegenerate points $(P, I)$ with $I(1)=I_{0}(1)$ and also to points with $I(1)=I_{0}(1)+2$.) In this case $P_{\tau_{i}}$ is a rotation through a small angle $t$, with eigenvalues $e^{ \pm i t}$, and with $\rho$-index as in Figure 2.

Note that $P_{\tau_{i}} \rightarrow P_{\sigma^{k}}$ as $t \rightarrow 0$ and that $I_{\tau_{i}}=I_{\sigma^{k}}$ away from $\rho=1$.

We can choose the lengths $L(\sigma, i, t)=$ length $\tau_{i}(\sigma, t)$ to be any continuous functions of $t$ with

$$
L(\sigma, i, 0)=m_{i} L(\sigma)
$$

and so that for all $\sigma, \sigma^{\prime}, i, j, m, n$ there is an $\varepsilon>0$ so that, if $t<\varepsilon$,

$$
L\left(\sigma^{m m_{i}(\sigma)}\right)<L\left(\sigma^{\prime n m j\left(\sigma^{\prime}\right)} \Longrightarrow m L(\sigma, i, t)<n L\left(\sigma^{\prime}, j, t\right)\right.
$$


and so that, for each $\sigma$ and each $t$, the $L(\sigma, i, t)$ have the same order relations as the critical values of the perturbed function on the characteristic manifold.

Corollary 4 is true in the nondegenerate case due to the lemma and Bott's formula (1) for the index of the iterates; if any iterate of a nondegenerate closed geodesic $\tau$ has even index, then $\tau$ must be hyperbolic, in which case $\operatorname{Index}\left(\tau^{m}\right)=m \operatorname{Index}(\tau)$. Note that $\mathbb{M}_{2 p}$ may also contain $D_{\tau}$ with $\operatorname{mult}(\tau)=2 p$; however in this case $\tau \in \mathfrak{G}^{-}$, so that $D_{\tau} \in \operatorname{Ker} \delta$.

The corollary follows as well in the degenerate case: if $\tau(\sigma)$ is a limiting phantom geodesic, and if some iterate of $\tau$ has even index, then $\tau$ must be a limit of hyperbolic phantom geodesics, in which case again $\operatorname{Index}\left(\tau^{m}\right)=m \operatorname{Index}(\tau)$.

It seems that one could do the construction of Proposition II without much more difficulty for a manifold of dimenson greater than 2 ; however, there seem to be real problems with Proposition I in higher dimensions. Here is a simple case of the difficulty we expect: Suppose $\sigma$ and $\tau$ are prime closed geodesics, with $\operatorname{Index}(\sigma)=\operatorname{Index}(\tau)+1, d D_{\sigma}=D_{\tau}$, but with $\operatorname{Index}\left(\tau^{p}\right) \geq \operatorname{Index}\left(\sigma^{p}\right)$ for some prime $p$. If this occurs (and we see no reason why it should not), then it is impossible to make the attaching map for $D_{\sigma^{p}}$ both cellular and equivariant, since the fixed point set of the action of $\mathbb{Z}_{p}$ on $D_{\sigma^{p}}$ is the disk $\left(D_{\sigma}\right)^{p} \subseteq D_{\sigma^{p}}$.

\section{REFERENCES}

1. R. Bott, On the iteration of closed geodesics and the Sturm intersection theory, Comm. Pure Appl. Math. 9 (1956), 171-206. MR 19:859f

2. M. Greenberg, Lectures on Aglebraic Topology, Benjamin, Reading MA, 1967. MR 35:6137

3. D. Gromoll and W. Meyer, Periodic geodesics on compact Riemannian manifolds, J. Diff. Geom. 3 (1969), 493-510. MR 41:9143

4. N. Hingston, An equivariant model for the free loop space of $S^{N}$, American J. of Math. 114 (1991), 139-155. MR 93b:55011

5. N. Hingston, Curve shortening, equivariant Morse theory and closed geodesics on the 2sphere, A.M.S. Proceedings of Symposia in Pure Mathematics, vol. 54, Amer. Math. Soc., Providence, 1993, pp. 423-429. MR 94a:00012

6. N. Hingston, On the growth of the number of closed geodesics on the two-sphere, IMRN 9 (No. 4) (1993), 253-262. MR 94m:58044

7. J. D. Jones, Cyclic homology and equivariant homology, Inventiones Math. 87 (1987), 403424. MR 88f: 18016

8. W. Klingenberg, Lectures on Closed Geodesics, Springer-Verlag, Berlin, 1978. MR 57:17563

9. W. Klingenberg, Riemannian Geometry, de Gruyter, Berlin, 1982. MR 84j:53001

10. G. Lodder, Dihedral homology and the free loop space, Proc. London Math. Soc. 360 (1) (1990), 201-224. MR 91a:55007

11. M. Morse, The Calculus of Variations in the Large, Amer. Math. Soc. Colloq. Publ., vol. 18, Amer. Math. Soc., Providence, 1934.

12. H. B. Rademacher, On the equivariant Morse chain complex on the space of closed curves., Math. Z. 201 (1989), 279-302. MR 90i:58027

Department of Mathematics, The College of New Jersey, P. O. Box 7718, Ewing, NEW JERSEY 08628-0718

E-mail address: hingston@tcnj.edu 